Article

\title{
Evaluation Based on Distance from Average Solution Method for Multiple Criteria Group Decision Making under Picture 2-Tuple Linguistic Environment
}

\author{
Siqi Zhang ${ }^{1}$, Hui Gao ${ }^{1}$, Guiwu Wei ${ }^{1}{ }^{\circledR}$, Yu Wei ${ }^{2, *(1)}$ and Cun Wei ${ }^{1,3}$ \\ 1 School of Business, Sichuan Normal University, Chengdu 610101, China; zsq1277@163.com (S.Z.); \\ gaohuisxy@sicnu.edu.cn (H.G.); weiguiwu1973@sicnu.edu.cn (G.W.); weicun1990@163.com (C.W.) \\ 2 School of Finance, Yunnan University of Finance and Economics, Kunming 650221, China \\ 3 School of Science, Southwest Petroleum University, Chengdu 610500, China \\ * Correspondence: weiyusy@126.com or ywei@home.swjtu.edu.cn
}

Received: 31 January 2019; Accepted: 5 March 2019; Published: 8 March 2019

\begin{abstract}
In this paper, we design the EDAS (evaluation based on distance from average solution) model with picture 2-tuple linguistic numbers (P2TLNs). First, we briefly reviewed the definition of P2TLSs and introduced the score function, accuracy function, and operational laws of P2TLNs. Then, we combined the traditional EDAS model for multiple criteria group decision making (MCGDM) with P2TLNs. Our presented model was more accurate and effective for considering the conflicting attributes. Finally, a numerical case for green supplier selection was given to illustrate this new model, and some comparisons were also conducted between the picture 2-tuple linguistic weighted averaging (P2TLWA), picture 2-tuple linguistic weighted geometric (P2TLWG) aggregation operators and EDAS model with P2TLNs, to further illustrate the advantages of the new method.
\end{abstract}

Keywords: multiple criteria group decision making (MCGDM) problems; picture fuzzy sets (PFSs); picture 2-tuple linguistic numbers (P2TLNs); picture 2-tuple linguistic sets (P2TLSs); EDAS model; green supplier selection

\section{Introduction}

The traditional EDAS (evaluation based on distance from average solution) method [1], which can consider the conflicting attributes, has been studied in many multi-attribute decision making (MADM) problems. By computing the average solution (AV), this model can describe the difference between all the alternatives and the AV based on two distance measures which are namely PDA (Positive Distance from Average) and NDA (Negative Distance from Average), the alternative with higher values of PDA and lower values of NDA is the best choice. Until now, lots of MADM methods such as the VIKOR (VIseKriterijumska Optimizacija I KOmpromisno Resenje) method [2,3], the ELECTRE (ELimination and Choice Expressing the Reality) method [4], the TOPSIS (Technique for Order Preference by Similarity to Ideal Solution) method [5], the PROMETHEE (Preference Ranking Organisation Method for Enrichment Evaluations) method [6,7], the GRA (Grey relational Analysis) method [8], the MULTIMOORA method [9] and the TODIM (an acronym in Portuguese for Interactive Multi-Criteria Decision Making) method [10-12] were broadly investigated by a large amount of scholars. Compared to the existing work, the EDAS model owns the merit of only taking AVs into account with respect to the intangibility of decision maker (DM) and the uncertainty of the decision making environment to obtain more accurate and effective aggregation results.

Atanassov [13] introduced the concept of intuitionistic fuzzy sets (IFSs), which is a generalization of the concept of fuzzy sets [14]. Atanassov and Gargov [15], and Atanassov [16] proposed the concept 
of interval-valued intuitionistic fuzzy sets (IVIFSs), which are characterized by a membership function, a non-membership function, and a hesitancy function whose values are intervals. Recently, Cuong and Kreinovich [17] proposed picture fuzzy sets (PFSs) and investigated some basic operations and properties of PFSs. The PFS is characterized by three functions expressing the degree of membership, the degree of neutral membership, and the degree of non-membership. The only constraint is that the sum of the three degrees must not exceed 1. Singh [18] presented the geometrical interpretation of PFSs and proposed correlation coefficients for PFSs. Son [19] presented a novel distributed picture fuzzy clustering (FC) method on PFSs. Thong and Son [20] proposed the model between picture FC and intuitionistic fuzzy recommender systems for medical diagnosis. Thong and Son [21] proposed automatic picture fuzzy clustering (AFC-PFS) for determining the most suitable number of clusters for AFC-PFS. Wei [22] proposed the MADM method based on the proposed picture fuzzy cross entropy. Son [23] defined the generalized picture distance measures and picture association measures. Son and Thong [24] developed some novel hybrid forecast models with picture FC for weather nowcasting from satellite image sequences. Wei [25] gave some cosine similarity measures of PFSs for strategic decision making on the basis of traditional similarity measures [26,27]. Wei [28] proposed some aggregation operators for MADM based on the PFSs based on traditional aggregation operators [29-35]. Wei [36] defined some similarity measures for PFSs. Wei [12] proposed the TODIM method for picture fuzzy MADM. Wei and Gao [37] developed the generalized dice similarity measures for PFSs. Wei [38] proposed some picture fuzzy Hamacher aggregation operators in MADM with traditional Hamacher operations [39-42]. Wei et al. [43] designed the projection models for MADM with picture fuzzy information. Wei et al. [44] proposed some picture 2-tuple linguistic operators in MADM. Wei [45] proposed some Bonferroni mean (BM) operators with P2TLNs in MADM. Wei [46] defined some picture uncertain linguistic BM operators for MADM.

Turskis et al. [1] originally defined the EDAS method for multi-criteria inventory classification. Keshavarz Ghorabaee et al. [47] proposed the extended EDAS method for supplier selection. Kahraman et al. [48] established the EDAS model under intuitionistic fuzzy information for solid waste disposal site selection. Keshavarz Ghorabaee et al. [49] extended the EDAS method with interval type-2 fuzzy sets. Keshavarz Ghorabaee et al. [50] defined the multi-criteria EDAS model with interval type-2 fuzzy sets. Keshavarz Ghorabaee et al. [51] proposed the stochastic EDAS method for MADM with normally distributed data. Peng and Liu [52] resolved the neutrosophic soft decision making method based on EDAS. Ecer [53] gave third-party logistics provider selection with the fuzzy AHP and the fuzzy EDAS integrated method. Feng et al. [54] developed the EDAS method for hesitant fuzzy linguistic MADM. Ilieva [55] assigned the group decision models with EDAS for interval fuzzy sets. Karasan and Kahraman [56] defined the interval-valued neutrosophic EDAS method. Keshavarz-Ghorabaee et al. [57] developed the dynamic fuzzy EDAS method for multi-criteria subcontractor evaluation. Stevic et al. [58] gave the selection of carpenter manufacturer using the fuzzy EDAS method. Keshavarz-Ghorabaee et al. [59] gave a comparative analysis of the rank reversal phenomenon with the EDAS and TOPSIS methods.

Wei et al. [44] introduced the concept of P2TLSs based on PFSs [17] and the 2-tuple linguistic information processing model [60], and developed some BM and geometric BM operators with P2TLNs. However, no studies using the EDAS model with P2TLNs were found in the literature. Hence, it was necessary to take the picture 2-tuple linguistic EDAS model into account. The purpose of our work is to establish an extended EDAS model according to the traditional EDAS method and P2TLNs to study multiple criteria group decision making (MCGDM) problems more effectively. Thus, the main contributions of this paper are (1) to extend EDAS models to picture 2-tuple linguistic sets; (2) to combine the traditional EDAS model for MCGDM with P2TLNs; (3) to provide a numerical case for green supplier selection to illustrate this new model and conduct some comparisons between the EDAS model with P2TLNs, and P2TLWA and P2TLWG aggregation operators to further illustrate advantages of the new method. 
The structure of our paper is organized as follows: definition, score function, accuracy function, and operational formulas of P2TLNs are briefly introduced in Section 2. We introduce some aggregation operators of P2TLNs in Section 3. We combine the traditional EDAS model for MCGDM with P2TLNs, and the computing steps are simply depicted in Section 4. In Section 5, a numerical example for green supplier selection has been given to illustrate this new model, and some comparisons between the use of P2TLWA and P2TLWG operators in the EDAS model with P2TLNs were also conducted to further illustrate the advantages of the new method. Section 6 describes some conclusions of our work.

\section{Preliminaries}

In the following, we introduced some basic concepts related to 2-tuple linguistic term sets and PFSs.

\subsection{2-Tuple Linguistic Term Sets}

Let $S=\left\{s_{i} \mid i=1,2, \cdots, t\right\}$ be a linguistic term set with odd cardinality. $s_{i}$ represents a possible value for a linguistic variable, and it should satisfy the following characteristics [61]:

(1) The set is ordered: $s_{i}>s_{j}$, if $i>j$; (2) Max operator: $\max \left(s_{i}, s_{j}\right)=s_{i}$, if $s_{i} \geq s_{j}$; (3) Min operator: $\min \left(s_{i}, s_{j}\right)=s_{i}$, if $s_{i} \leq s_{j}$. For example, $S$ can be defined as

$$
\begin{gathered}
S=\left\{s_{1}=\text { extremely poor, } s_{2}=\text { very poor }, s_{3}=\text { poor }, s_{4}=\right.\text { medium, } \\
\left.s_{5}=\text { good, } s_{6}=\text { very good, } s_{7}=\text { extremely good }\right\}
\end{gathered}
$$

Herrera and Martinez [60] defined the 2-tuple fuzzy linguistic representation model based on the concept of symbolic translation. It is utilized for depicting the linguistic information with a 2-tuple $\left(s_{i}, \alpha_{i}\right)$, where $s_{i}$ is a linguistic label from predefined linguistic term set $S$, and $\alpha_{i}$ is the value of symbolic translation, and $\alpha_{i} \in[-0.5,0.5)$.

\subsection{Picture Fuzzy Sets (PFSs)}

Definition 1 ([17]). A PFS on the universe. X is an object of the form

$$
A=\left\{\left\langle x, \mu_{A}(x), \eta_{A}(x), v_{A}(x)\right\rangle \mid x \in X\right\}
$$

where $\mu_{A}(x) \in[0,1]$ is called the "degree of positive membership of $A$ ", $\eta_{A}(x) \in[0,1]$ is defined as the "degree of neutral membership of $A^{\prime \prime}$, and $v_{A}(x) \in[0,1]$ is defined as the "degree of negative membership of $A$ ", and $\mu_{A}(x), \eta_{A}(x), v_{A}(x)$ satisfy the following condition: $0 \leq \mu_{A}(x)+\eta_{A}(x)+v_{A}(x) \leq 1, \forall x \in X$. Then, for $x \in X, \pi_{A}(x)=1-\left(\mu_{A}(x)+\eta_{A}(x)+v_{A}(x)\right)$ could be defined as the degree of refusal membership of $x$ in $A$.

Definition 2 ([17]). Let $\alpha=\left(\mu_{\alpha}, \eta_{\alpha}, v_{\alpha}\right)$ and $\beta=\left(\mu_{\beta}, \eta_{\beta}, v_{\beta}\right)$ be two PFNs, the operation formula of them can be given:

(1) $\quad \alpha \oplus \beta=\left(\mu_{\alpha}+\mu_{\beta}-\mu_{\alpha} \mu_{\beta}, \eta_{\alpha} \eta_{\beta}, v_{\alpha} v_{\beta}\right)$;

(2) $\alpha \otimes \beta=\left(\mu_{\alpha} \mu_{\beta}, \eta_{\alpha}+\eta_{\beta}-\eta_{\alpha} \eta_{\beta}, v_{\alpha}+v_{\beta}-v_{\alpha} v_{\beta}\right)$;

(3) $\lambda \alpha=\left(1-\left(1-\mu_{\alpha}\right)^{\lambda}, \eta_{\alpha}^{\lambda}, v_{\alpha}^{\lambda}\right), \lambda>0$;

(4) $\alpha^{\lambda}=\left(\mu_{\alpha}^{\lambda}, 1-\left(1-\eta_{\alpha}\right)^{\lambda}, 1-\left(1-v_{\alpha}\right)^{\lambda}\right), \lambda>0$.

According to Definition 2, the operation laws have the following properties [17].

$$
\alpha \oplus \beta=\beta \oplus \alpha, \alpha \otimes \beta=\beta \otimes \alpha,\left((\alpha)^{\lambda_{1}}\right)^{\lambda_{2}}=(\alpha)^{\lambda_{1} \lambda_{2}}
$$




$$
\begin{gathered}
\lambda(\alpha \oplus \beta)=\lambda \alpha \oplus \lambda \beta,(\alpha \otimes \beta)^{\lambda}=(\alpha)^{\lambda} \otimes(\beta)^{\lambda} ; \\
\lambda_{1} \alpha \oplus \lambda_{2} \alpha=\left(\lambda_{1}+\lambda_{2}\right) \alpha,(\alpha)^{\lambda_{1}} \otimes(\alpha)^{\lambda_{2}}=(\alpha)^{\left(\lambda_{1}+\lambda_{2}\right)} .
\end{gathered}
$$

\subsection{Picture 2-Tuple Linguistic Sets (P2TLSs)}

In the following, we introduce the concepts and basic operations of the P2TLSs based on the PFSs [17] and 2-tuple linguistic information model [60].

Definition $3([44,45])$. A P2TLS $A$ in $X$ is given

$$
A=\left\{\left(s_{\theta(x)}, \rho\right),\left(\mu_{A}(x), \eta_{A}(x), v_{A}(x)\right), x \in X\right\},
$$

where $\left(s_{\theta(x)}, \rho\right) \in S, \rho \in[-0.5,0.5), u_{A}(x) \in[0,1], \eta_{A}(x) \in[0,1]$, and $v_{A}(x) \in[0,1]$, with the condition $0 \leq u_{A}(x)+\eta_{A}(x)+v_{A}(x) \leq 1, \forall x \in X, s_{\theta(a)} \in S$, and $\rho \in[-0.5,0.5)$. The numbers $\mu_{A}(x), \eta_{A}(x), v_{A}(x)$ represent, respectively, the degree of positive membership, degree of negative membership, and degree of negative membership of the element $x$ to 2-tuple linguistic variable $\left(s_{\theta(x)}, \rho\right)$.

For convenience, we call $\widetilde{\alpha}=<\left(s_{\theta(a)}, \rho\right),(u(a), \eta(a), v(a))>$ a P2TLN, where $\mu_{\alpha} \in[0,1], \eta_{\alpha} \in$ $[0,1], v_{\alpha} \in[0,1], \mu_{\alpha}+\eta_{\alpha}+v_{\alpha} \leq 1, s_{\theta(a)} \in S$ and $\rho \in[-0.5,0.5)$.

Definition 4 ([44]). Let $\widetilde{a}=<\left(s_{\theta(a)}, \rho\right),(u(a), \eta(a), v(a))>$ be a P2TLN, and a score function $\widetilde{a}$ can be defined as follows:

$$
S(\widetilde{a})=\Delta\left(\Delta^{-1}\left(s_{\theta(a)}, \rho\right) \cdot \frac{1+\mu_{\alpha}-v_{\alpha}}{2}\right), \Delta^{-1}(S(\widetilde{a})) \in[1, t] .
$$

Definition 5 ([44]). Let $\widetilde{a}=<\left(s_{\theta(a)}, \rho\right),(u(a), \eta(a), v(a))>$ be a P2TLN, and the accuracy function can be defined as follows:

$$
H(\widetilde{a})=\Delta\left(\Delta^{-1}\left(s_{\theta(a)}, \rho\right) \cdot \frac{\mu_{\alpha}+\eta_{\alpha}+v_{\alpha}}{2}\right), \Delta^{-1}(H(\widetilde{a})) \in[1, t]
$$

Definition 6 ([44]). Let $\widetilde{a}_{1}=<\left(s_{\theta\left(a_{1}\right)}, \rho_{1}\right),\left(u\left(a_{1}\right), \eta\left(a_{1}\right), v\left(a_{1}\right)\right)>$ and $\widetilde{a}_{2}=<\left(s_{\theta\left(a_{2}\right)}, \rho_{2}\right)$, $\left(u\left(a_{2}\right), \eta\left(a_{2}\right), v\left(a_{2}\right)\right)>$ be two P2TLNs, $S\left(\widetilde{a}_{1}\right)=\Delta\left(\Delta^{-1}\left(s_{\theta\left(a_{1}\right)}, \rho_{1}\right) \cdot \frac{1+\mu_{\alpha_{1}}-v_{\alpha_{1}}}{2}\right)$ and $S\left(\widetilde{a}_{2}\right)=$ $\Delta\left(\Delta^{-1}\left(s_{\theta\left(a_{2}\right)}, \rho_{2}\right) \cdot \frac{1+\mu_{\alpha_{2}}-v_{\alpha_{2}}}{2}\right)$ be the scores of $\widetilde{a}_{1}$ and $\tilde{a}_{2}$, respectively, and let $H\left(\widetilde{a}_{1}\right)=$ $\Delta\left(\Delta^{-1}\left(s_{\theta\left(a_{1}\right)}, \rho_{1}\right) \cdot \frac{\mu_{\alpha_{1}}+\eta_{\alpha_{1}}+v_{\alpha_{1}}}{2}\right)$ and $H\left(\widetilde{a}_{2}\right)=\Delta\left(\Delta^{-1}\left(s_{\theta\left(a_{2}\right)}, \rho_{2}\right) \cdot \frac{\mu_{\alpha_{2}}+\eta_{\alpha_{2}}+v_{\alpha_{2}}}{2}\right)$ be the accuracy degrees of $\widetilde{a}_{1}$ and $\widetilde{a}_{2}$, respectively, then if $S\left(\widetilde{a}_{1}\right)<S\left(\widetilde{a}_{2}\right), \widetilde{a}_{1}<\widetilde{a}_{2}$; if $S\left(\widetilde{a}_{1}\right)=S\left(\widetilde{a}_{2}\right)$, then (1) if $H\left(\widetilde{a}_{1}\right)=H\left(\widetilde{a}_{2}\right)$, then $\widetilde{a}_{1}=\widetilde{a}_{2} ;(2)$ if $H\left(\widetilde{a}_{1}\right)<H\left(\widetilde{a}_{2}\right)$, then, $\widetilde{a}_{1}<\widetilde{a}_{2}$.

Some operational laws of P2TLNs are defined as follows: 
Definition 7 ([44]). Let $\tilde{a}_{1}=<\quad\left(s_{\theta\left(a_{1}\right)}, \rho_{1}\right),\left(u\left(a_{1}\right), \eta\left(a_{1}\right), v\left(a_{1}\right)\right) \quad>$ and $\tilde{a}_{2}=<$ $\left(s_{\theta\left(a_{2}\right)}, \rho_{2}\right),\left(u\left(a_{2}\right), \eta\left(a_{2}\right), v\left(a_{2}\right)\right)>$ be two P2TLNs, then

$$
\begin{aligned}
& \tilde{a}_{1} \oplus \tilde{a}_{2}=\left\langle\Delta\left(\Delta^{-1}\left(s_{\theta\left(a_{1}\right)}, \rho_{1}\right)+\Delta^{-1}\left(s_{\theta\left(a_{2}\right)}, \rho_{2}\right)\right),\right. \\
& \left.\left(u\left(a_{1}\right)+u\left(a_{2}\right)-u\left(a_{1}\right) u\left(a_{2}\right), \eta\left(a_{1}\right) \eta\left(a_{2}\right), v\left(a_{1}\right) v\left(a_{2}\right)\right)\right\rangle ; \\
& \widetilde{a}_{1} \otimes \widetilde{a}_{2}=\left\langle\Delta\left(\Delta^{-1}\left(s_{\theta\left(a_{1}\right)}, \rho_{1}\right) \cdot \Delta^{-1}\left(s_{\theta\left(a_{2}\right)}, \rho_{2}\right)\right),\right. \\
& \left.\left(u\left(a_{1}\right) u\left(a_{2}\right), \eta\left(a_{1}\right)+\eta\left(a_{2}\right)-\eta\left(a_{1}\right) \eta\left(a_{2}\right), v\left(a_{1}\right)+v\left(a_{2}\right)-v\left(a_{1}\right) v\left(a_{2}\right)\right)\right\rangle \text {; } \\
& \lambda \widetilde{a}_{1}=\left\langle\Delta\left(\lambda \Delta^{-1}\left(s_{\theta\left(a_{1}\right)}, \rho_{1}\right)\right),\left(1-\left(1-u\left(a_{1}\right)\right)^{\lambda}, \eta\left(a_{1}\right)^{\lambda}, v\left(a_{1}\right)^{\lambda}\right)\right\rangle ; \\
& \left(\widetilde{a}_{1}\right)^{\lambda}=\left\langle\Delta\left(\left(\Delta^{-1}\left(s_{\theta\left(a_{1}\right)}, \rho_{1}\right)\right)^{\lambda}\right),\left(u\left(a_{1}\right)^{\lambda}, 1-\left(1-\eta\left(a_{1}\right)\right)^{\lambda}, 1-\left(1-v\left(a_{1}\right)\right)^{\lambda}\right)\right\rangle .
\end{aligned}
$$

\section{Picture 2-Tuple Linguistic Aggregation Operators}

In this section, we propose some aggregation operators with P2TLNs, such as the P2TLWA operator and the P2TLWG operator.

Definition 8. Let $\widetilde{\alpha}_{j}=\left\langle\left(s_{j}, \rho_{j}\right),\left(\mu_{j}, \eta_{j}, v_{j}\right)\right\rangle(j=1,2, \cdots, n)$ be a collection of P2TLNs, and the P2TLWA operator can be represented as

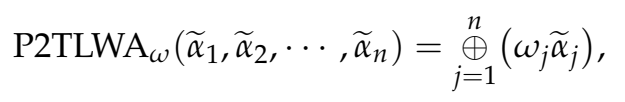

where $\omega=\left(\omega_{1}, \omega_{2}, \ldots, \omega_{n}\right)^{T}$ is the weight vector of $\widetilde{\alpha}_{j}(j=1,2, \ldots, n)$ and $\omega_{j}>0, \sum_{j=1}^{n} \omega_{j}=1$.

Based on the Definition 8, we can get the following result:

Theorem 1. The aggregated value by using the P2TLWA operator is also a P2TLN, where

$$
\begin{aligned}
& \operatorname{P} \operatorname{TLWA}_{\omega}\left(\widetilde{\alpha}_{1}, \widetilde{\alpha}_{2}, \cdots, \widetilde{\alpha}_{n}\right)=\bigoplus_{j=1}^{n}\left(\omega_{j} \widetilde{\alpha}_{j}\right) \\
& =\left\langle\Delta\left(\sum_{j=1}^{n} \omega_{j} \Delta^{-1}\left(s_{j}, \rho_{j}\right)\right),\left(1-\prod_{j=1}^{n}\left(1-\mu_{j}\right)^{\omega_{j}}, \prod_{j=1}^{n}\left(\eta_{j}\right)^{\omega_{j}}, \prod_{j=1}^{n}\left(v_{j}+\eta_{j}\right)^{\omega_{j}}-\prod_{j=1}^{n}\left(\eta_{j}\right)^{\omega_{j}}\right)\right\rangle
\end{aligned}
$$

where $\omega=\left(\omega_{1}, \omega_{2}, \ldots, \omega_{n}\right)^{T}$ is the weight vector of $\widetilde{\alpha}_{j}(j=1,2, \ldots, n)$ and $\omega_{j}>0, \sum_{j=1}^{n} \omega_{j}=1$.

Definition 9. Let $\widetilde{\alpha}_{j}=\left\langle\left(s_{j}, \rho_{j}\right),\left(\mu_{j}, \eta_{j}, v_{j}\right)\right\rangle(j=1,2, \cdots, n)$ be a collection of P2TLNs, the P2TLWG operator can be represented as

$$
\operatorname{P} \mathrm{TLWG}_{\omega}\left(\widetilde{\alpha}_{1}, \widetilde{\alpha}_{2}, \cdots, \widetilde{\alpha}_{n}\right)=\bigotimes_{j=1}^{n}\left(\widetilde{\alpha}_{j}\right)^{\omega_{j}}
$$

where $\omega=\left(\omega_{1}, \omega_{2}, \ldots, \omega_{n}\right)^{T}$ is the weight vector of $\widetilde{\alpha}_{j}(j=1,2, \ldots, n)$ and $\omega_{j}>0, \sum_{j=1}^{n} \omega_{j}=1$.

Based on Definition 9, we can get the following result: 
Theorem 2. The aggregated value by using the P2TLWG operator is also a P2TLN, where

$$
\begin{aligned}
& \text { P2TLWG }_{\omega}\left(\widetilde{\alpha}_{1}, \widetilde{\alpha}_{2}, \cdots, \widetilde{\alpha}_{n}\right)=\bigotimes_{j=1}^{n}\left(\widetilde{\alpha}_{j}\right)^{\omega_{j}} \\
& =\left\langle\Delta\left(\prod_{j=1}^{n}\left(\Delta^{-1}\left(s_{j}, \rho_{j}\right)^{\omega_{j}}\right)\right),\left(\prod_{j=1}^{n}\left(\mu_{\alpha_{j}}+\eta_{\alpha_{j}}\right)^{\omega_{j}}-\prod_{j=1}^{n}\left(\eta_{\alpha_{j}}\right)^{\omega_{j}}, \prod_{j=1}^{n}\left(\eta_{\alpha_{j}}\right)^{\omega_{j}}, 1-\prod_{j=1}^{n}\left(1-v_{\alpha_{j}}\right)^{\omega_{j}}\right)\right\rangle
\end{aligned}
$$

where $\omega=\left(\omega_{1}, \omega_{2}, \ldots, \omega_{n}\right)^{T}$ is the weight vector of $\alpha_{j}(j=1,2, \ldots, n)$ and $\omega_{j}>0, \sum_{j=1}^{n} \omega_{j}=1$.

\section{The EDAS Model with P2TLNs}

The traditional EDAS method [1], which can consider the conflicting attributes, has been studied in many MCDM problems. By computing the average solution (AV), this model can describe the difference between all the alternatives and the AV based on two distance measures which are namely PDA (positive distance from average) and NDA (negative distance from average); the alternative with higher values of PDA and lower values of PDA is the best choice. To combine the EDAS model with P2TLNs, we construct the EDAS model so the evaluation values are presented by P2TLNs. The computing steps of our proposed model can be established as follows.

Suppose there are $m$ alternatives $\left\{\delta_{1}, \delta_{2}, \ldots \delta_{m}\right\}, n$ attributes $\left\{G_{1}, G_{2}, \ldots G_{n}\right\}$, and $r$ experts $\left\{a_{1}, a_{2}, \ldots a_{r}\right\}$, let $\left\{\omega_{1}, \omega_{2}, \ldots \omega_{n}\right\}$ and $\left\{\theta_{1}, \theta_{2}, \ldots \theta_{r}\right\}$ be the attribute's weighting vector and expert's weighting vector which satisfy $\omega_{i} \in[0,1], \theta_{i} \in[0,1]$ and $\sum_{i=1}^{n} \omega_{i}=1, \sum_{i=1}^{t} \theta_{i}=1$. Then:

Step 1. Construct the picture 2-tuple linguistic decision matrix $\widetilde{R}=\left(\widetilde{r}_{i j}\right)_{m \times n}=$ $\left\langle\left(s_{i j}, \rho_{i j}\right),\left(\mu_{i j}, \eta_{i j}, v_{i j}\right)\right\rangle_{m \times n^{\prime}} i=1,2, \ldots, m, j=1,2, \ldots, n$, which can be depicted as follows.

$$
\widetilde{R}=\left(\widetilde{r}_{i j}\right)_{m \times n}=\left[\begin{array}{cccc}
\widetilde{r}_{11} & \widetilde{r}_{12} & \ldots & \widetilde{r}_{1 n} \\
\widetilde{r}_{21} & \widetilde{r}_{22} & \ldots & \widetilde{r}_{2 n} \\
\vdots & \vdots & \vdots & \vdots \\
\widetilde{r}_{m 1} & \widetilde{r}_{m 2} & \ldots & \widetilde{r}_{m n}
\end{array}\right],
$$

where $\widetilde{r}_{i j}$ denotes the P2TLNs of alternative $\vartheta_{i}$ on attribute $U_{j}$ by expert $q^{r}$.

Step 2. Normalize the evaluation matrix $\widetilde{R}=\left(\widetilde{r}_{i j}\right)_{m \times n}$ to $\widetilde{R}^{\prime}=\left(\widetilde{r}_{i j}^{\prime}\right)_{m \times n}$.

For benefit attributes:

$$
\widetilde{r}_{i j}^{\prime}=\widetilde{r}_{i j}=\left\langle\left(s_{i j}, \rho_{i j}\right),\left(\mu_{i j}, \eta_{i j}, v_{i j}\right)\right\rangle, i=1,2, \ldots, m, j=1,2, \ldots, n
$$

For cost attributes:

$$
\widetilde{r}_{i j}^{\prime}=\left(\widetilde{r}_{i j}\right)^{c}=\left\langle\Delta\left(T-\Delta^{-1}\left(s_{i j}, \rho_{i j}\right)\right),\left(v_{i j}, \eta_{i j}, \mu_{i j}\right)\right\rangle, i=1,2, \ldots, m, j=1,2, \ldots, n .
$$

Step 3. According to the decision making matrix $\widetilde{R}^{\prime}=\left(\widetilde{r}_{i j}^{\prime}\right)_{m \times n}$ and expert's weighting vector $\left\{\delta_{1}, \delta_{2}, \ldots \delta_{r}\right\}$, we can utilize overall ${\widetilde{r^{\prime}}}_{i j}$ to $r^{\prime}{ }_{i j}$ by using P2TLWA or P2TLWG aggregation operators, and the computing results can be presented as follows.

$$
R=\left[r_{i j}^{\prime}\right]_{m \times n}=\left[\begin{array}{cccc}
r_{11}^{\prime} & r^{\prime}{ }_{12} & \ldots & r^{\prime}{ }_{1 n} \\
r_{21}^{\prime} & r^{\prime}{ }_{22} & \ldots & r_{2 n}^{\prime} \\
\vdots & \vdots & \vdots & \vdots \\
r^{\prime}{ }_{m 1} & r^{\prime}{ }_{m 2} & \ldots & r_{m n}^{\prime}
\end{array}\right]
$$


Step 4. Compute the value of AV based on all proposed attributes;

$$
\mathrm{AV}=\left[\mathrm{AV}_{j}\right]_{1 \times n}=\left[\frac{\sum_{i=1}^{m} r^{\prime}{ }_{i j}}{m}\right]_{1 \times n} .
$$

Based on Definition 8,

$$
\begin{gathered}
\sum_{i=1}^{m} r^{\prime}{ }_{i j}=\left\langle\Delta\left(\sum_{i=1}^{m} \Delta^{-1}\left(s_{i}, \rho_{i}\right)\right),\left(1-\prod_{i=1}^{m}\left(1-\mu_{i j}^{\prime}\right), \prod_{i=1}^{m} \eta_{i j}^{\prime}, \prod_{i=1}^{m}\left(v_{i j}^{\prime}+\eta_{i j}^{\prime}\right)-\prod_{i=1}^{m} \eta_{i j}^{\prime}\right)\right\rangle \\
\mathrm{AV}=\left[\mathrm{AV}_{j}\right]_{1 \times n}=\left[\frac{\sum_{i=1}^{m} r^{\prime}{ }^{\prime} i}{m}\right]_{1 \times n} \\
=\left\langle\Delta\left(\sum_{i=1}^{m} \frac{1}{m} \Delta^{-1}\left(s_{i}, \rho_{i}\right)\right),\left(1-\prod_{i=1}^{m}\left(1-\mu_{i j}^{\prime}\right)^{\frac{1}{m}}, \prod_{i=1}^{m}\left(\eta^{\prime}{ }_{i j}\right)^{\frac{1}{m}}, \prod_{i=1}^{m}\left(v^{\prime}{ }_{i j}+\eta^{\prime}{ }_{i j}\right)^{\frac{1}{m}}-\prod_{i=1}^{m}\left(\eta^{\prime}{ }_{i j}\right)^{\frac{1}{m}}\right)\right\rangle
\end{gathered}
$$

Step 5. According to the results of AV, we can compute the PDA and NDA by using the following formula:

$$
\begin{aligned}
\mathrm{PDA}_{i j} & =\left[\mathrm{PDA}_{i j}\right]_{m \times n}=\frac{\max \left(0,\left(r^{\prime}{ }_{i j}-\mathrm{AV}_{j}\right)\right)}{\mathrm{AV}_{j}}, \\
\mathrm{NDA}_{i j} & =\left[\mathrm{NDA}_{i j}\right]_{m \times n}=\frac{\max \left(0,\left(\mathrm{AV}_{j}-r^{\prime}{ }_{i j}\right)\right)}{\mathrm{AV}_{j}} .
\end{aligned}
$$

For convenience, we can use the score function of P2TLNs presented in Definition 4 to determine the results of PDA and NDA as follows.

$$
\begin{aligned}
\mathrm{PDA}_{i j} & =\left[\mathrm{PDA}_{i j}\right]_{m \times n}=\frac{\max \left(0,\left(s\left(r_{i j}^{\prime}\right)-s\left(\mathrm{AV}_{j}\right)\right)\right)}{s\left(\mathrm{AV}_{j}\right)} \\
\mathrm{NDA}_{i j} & =\left[\mathrm{NDA}_{i j}\right]_{m \times n}=\frac{\max \left(0,\left(s\left(\mathrm{AV}_{j}\right)-s\left(r^{\prime}{ }_{i j}\right)\right)\right)}{s\left(\mathrm{AV}_{j}\right)}
\end{aligned}
$$

Step 6. Calculate the values of $\mathrm{SP}_{i}$ and $\mathrm{SN}_{i}$ which denotes the weighted sum of PDA and NDA, the computing formula are provided as follows.

$$
\mathrm{SP}_{i}=\sum_{j=1}^{n} w_{j} P D A_{i j}, \mathrm{SN}_{i}=\sum_{j=1}^{n} w_{j} N D A_{i j}
$$

Step 7. The results of Equation (23) can be normalized as

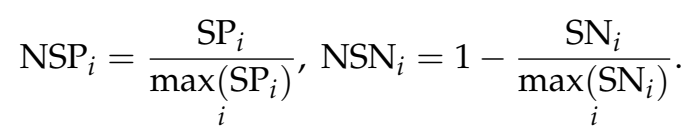

Step 8. Compute the values of appraisal score (AS) based on each alternative's $\mathrm{NSP}_{i}$ and $\mathrm{NSN}_{i}$.

$$
\mathrm{AS}_{i}=\frac{1}{2}\left(\mathrm{NSP}_{i}+\mathrm{NSN}_{i}\right)
$$

Step 9. According to the calculating results of the AS, we can rank all the alternatives; the bigger the value of AS is, the better the selected alternative will be.

\section{The Numerical Example}

\subsection{Numerical for MCGDM Problems with PFNs}

In this section, we provide a numerical example for green supplier selection by using EDAS models with P2TLNs. Assuming that five possible green suppliers $\vartheta_{i}(i=1,2,3,4,5)$ are to be selected 
and there are four criteria to assess these green suppliers: (1) $U_{1}$ is the price factor; (2) $U_{2}$ is the delivery factor; (3) $\mathrm{U}_{3}$ is the environmental factors; (4) $\mathrm{U}_{4}$ is the product quality factor. The five possible green suppliers $\vartheta_{i}(i=1,2,3,4,5)$ are to be evaluated with P2TLNs with the four criteria by three experts, $a^{r}$ (attributes weight $\omega=(0.22,0.36,0.28,0.14)$, expert's weight $\delta=(0.24,0.45,0.31)$.).

Step 1. Construct the evaluation matrix $\widetilde{R}=\left(\widetilde{r}_{i j}\right)_{m \times n^{\prime}} i=1,2, \ldots, m, j=1,2, \ldots, n$ for each of the three experts, which are listed in Tables 1-3.

Table 1. Picture 2-tuple linguistic evaluation information by $q^{1}$.

\begin{tabular}{ccc}
\hline & $\mathbf{U}_{1}$ & $\mathbf{U}_{2}$ \\
\hline$\vartheta_{1}$ & $\left\langle\left(S_{3}, 0\right),(0.41,0.26,0.33)\right\rangle$ & $\left\langle\left(S_{5}, 0\right),(0.54,0.36,0.10)\right\rangle$ \\
$\vartheta_{2}$ & $\left\langle\left(S_{6}, 0\right),(0.72,0.11,0.17)\right\rangle$ & $\left\langle\left(S_{3}, 0\right),(0.25,0.17,0.58)\right\rangle$ \\
$\vartheta_{3}$ & $\left\langle\left(S_{1}, 0\right),(0.35,0.26,0.39)\right\rangle$ & $\left\langle\left(S_{2}, 0\right),(0.28,0.16,0.56)\right\rangle$ \\
$\vartheta_{4}$ & $\left\langle\left(S_{3}, 0\right),(0.47,0.22,0.31)\right\rangle$ & $\left\langle\left(S_{1}, 0\right),(0.16,0.38,0.46)\right\rangle$ \\
$\vartheta_{5}$ & $\left\langle\left(S_{5}, 0\right),(0.58,0.17,0.25)\right\rangle$ & $\left\langle\left(S_{3}, 0\right),(0.39,0.21,0.40)\right\rangle$ \\
\hline$\vartheta_{3}$ & $\mathbf{U}_{3}$ & $\mathbf{U}_{4}$ \\
\hline$\vartheta_{2}$ & $\left\langle\left(S_{1}, 0\right),(0.33,0.35,0.32)\right\rangle$ & $\left\langle\left(S_{2}, 0\right),(0.59,0.16,0.25)\right\rangle$ \\
$\vartheta_{3}$ & $\left\langle\left(S_{4}, 0\right),(0.59,0.15,0.26)\right\rangle$ & $\left\langle\left(S_{5}, 0\right),(0.68,0.21,0.11)\right\rangle$ \\
$\vartheta_{4}$ & $\left\langle\left(S_{7}, 0\right),(0.13,0.24,0.63)\right\rangle$ & $\left\langle\left(S_{3}, 0\right),(0.27,0.31,0.42)\right\rangle$ \\
$\vartheta_{5}$ & $\left\langle\left(S_{3}, 0\right),(0.56,0.19,0.25)\right\rangle$ & $\left\langle\left(S_{4}, 0\right),(0.41,0.29,0.30)\right\rangle$ \\
\hline
\end{tabular}

Table 2. Picture 2-tuple linguistic evaluation information by $q^{2}$.

\begin{tabular}{ccc}
\hline & $\mathbf{U}_{\mathbf{1}}$ & $\mathbf{U}_{\mathbf{2}}$ \\
\hline$\vartheta_{1}$ & $\left\langle\left(S_{2}, 0\right),(0.27,0.28,0.45)\right\rangle$ & $\left\langle\left(S_{1}, 0\right),(0.50,0.24,0.26)\right\rangle$ \\
$\vartheta_{2}$ & $\left\langle\left(S_{7}, 0\right),(0.59,0.17,0.24)\right\rangle$ & $\left\langle\left(S_{4}, 0\right),(0.66,0.21,0.13)\right\rangle$ \\
$\vartheta_{3}$ & $\left\langle\left(S_{2}, 0\right),(0.46,0.25,0.29)\right\rangle$ & $\left\langle\left(S_{3}, 0\right),(0.22,0.13,0.65)\right\rangle$ \\
$\vartheta_{4}$ & $\left\langle\left(S_{1}, 0\right),(0.34,0.10,0.56)\right\rangle$ & $\left\langle\left(S_{5}, 0\right),(0.34,0.42,0.24)\right\rangle$ \\
$\vartheta_{5}$ & $\left\langle\left(S_{5}, 0\right),(0.34,0.10,0.56)\right\rangle$ & $\left\langle\left(S_{4}, 0\right),(0.18,0.25,0.57)\right\rangle$ \\
\hline & $\mathbf{U}_{3}$ & $\mathbf{U}_{4}$ \\
\hline$\vartheta_{1}$ & $\left\langle\left(S_{4}, 0\right),(0.39,0.38,0.23)\right\rangle$ & $\left\langle\left(S_{3}, 0\right),(0.42,0.18,0.40)\right\rangle$ \\
$\vartheta_{2}$ & $\left\langle\left(S_{6}, 0\right),(0.60,0.16,0.24)\right\rangle$ & $\left\langle\left(S_{5}, 0\right),(0.75,0.10,0.15)\right\rangle$ \\
$\vartheta_{3}$ & $\left\langle\left(S_{3}, 0\right),(0.38,0.11,0.51)\right\rangle$ & $\left\langle\left(S_{4}, 0\right),(0.48,0.29,0.23)\right\rangle$ \\
$\vartheta_{4}$ & $\left\langle\left(S_{5}, 0\right),(0.29,0.31,0.40)\right\rangle$ & $\left\langle\left(S_{3}, 0\right),(0.57,0.25,0.18)\right\rangle$ \\
$\vartheta_{5}$ & $\left\langle\left(S_{2}, 0\right),(0.57,0.26,0.17)\right\rangle$ & $\left\langle\left(S_{1}, 0\right),(0.63,0.21,0.16)\right\rangle$ \\
\hline
\end{tabular}

Table 3. Picture 2-tuple linguistic evaluation information by $q^{3}$.

\begin{tabular}{ccc}
\hline & $\mathbf{U}_{\mathbf{1}}$ & $\mathbf{U}_{\mathbf{2}}$ \\
\hline$\vartheta_{1}$ & $\left\langle\left(S_{4}, 0\right),(0.19,0.33,0.48)\right\rangle$ & $\left\langle\left(S_{3}, 0\right),(0.32,0.29,0.39)\right\rangle$ \\
$\vartheta_{2}$ & $\left\langle\left(S_{5}, 0\right),(0.51,0.37,0.12)\right\rangle$ & $\left\langle\left(S_{5}, 0\right),(0.77,0.11,0.12)\right\rangle$ \\
$\vartheta_{3}$ & $\left\langle\left(S_{3}, 0\right),(0.59,0.25,0.16)\right\rangle$ & $\left\langle\left(S_{4}, 0\right),(0.35,0.25,0.40)\right\rangle$ \\
$\vartheta_{4}$ & $\left\langle\left(S_{7}, 0\right),(0.57,0.19,0.24)\right\rangle$ & $\left\langle\left(S_{3}, 0\right),(0.27,0.24,0.49)\right\rangle$ \\
$\vartheta_{5}$ & $\left\langle\left(S_{1}, 0\right),(0.22,0.21,0.57)\right\rangle$ & $\left\langle\left(S_{2}, 0\right),(0.41,0.36,0.23)\right\rangle$ \\
\hline & $\mathbf{U}_{3}$ & $\mathbf{U}_{4}$ \\
\hline$\vartheta_{1}$ & $\left\langle\left(S_{2}, 0\right),(0.59,0.24,0.17)\right\rangle$ & $\left\langle\left(S_{5}, 0\right),(0.74,0.16,0.10)\right\rangle$ \\
$\vartheta_{2}$ & $\left\langle\left(S_{4}, 0\right),(0.64,0.13,0.23)\right\rangle$ & $\left\langle\left(S_{7}, 0\right),(0.78,0.15,0.07)\right\rangle$ \\
$\vartheta_{3}$ & $\left\langle\left(S_{2}, 0\right),(0.49,0.17,0.34)\right\rangle$ & $\left\langle\left(S_{1}, 0\right),(0.53,0.28,0.19)\right\rangle$ \\
$\vartheta_{4}$ & $\left\langle\left(S_{1}, 0\right),(0.34,0.31,0.35)\right\rangle$ & $\left\langle\left(S_{3}, 0\right),(0.59,0.21,0.20)\right\rangle$ \\
$\vartheta_{5}$ & $\left\langle\left(S_{4}, 0\right),(0.71,0.19,0.10)\right\rangle$ & $\left\langle\left(S_{2}, 0\right),(0.34,0.42,0.24)\right\rangle$ \\
\hline
\end{tabular}

Step 2. Normalize the evaluation matrix $\widetilde{R}=\left[\widetilde{r}_{i j}\right]_{m \times n}$ to $\widetilde{R}^{\prime}=\left[\widetilde{r}_{i j}^{\prime}\right]_{m \times n}$; if all the attributes are benefitted, then normalization is not needed. 
Step 3. According to the decision making matrix $\widetilde{R}^{\prime}=\left[\widetilde{r}_{i j}^{\prime}\right]_{m \times n}$ and expert's weighting vector $\left\{\delta_{1}, \delta_{2}, \ldots \delta_{r}\right\}$, utilize overall $\widetilde{r}_{i j}^{\prime}$ to $r_{i j}^{\prime}$ by using the P2TLWA aggregation operator, and the computing results can be presented as follows in Table 4 .

Table 4. The fused values by using picture 2-tuple linguistic weighted averaging (P2TLWA) operator.

\begin{tabular}{ccc}
\hline & $\mathbf{U}_{\mathbf{1}}$ & $\mathbf{U}_{\mathbf{2}}$ \\
\hline$\vartheta_{1}$ & $\left\langle\left(S_{3},-0.1\right),(0.2836,0.2894,0.4269)\right\rangle$ & $\left\langle\left(S_{3},-0.4\right),(0.4609,0.2805,0.2586)\right\rangle$ \\
$\vartheta_{2}$ & $\left\langle\left(S_{6}, 0.1\right),(0.6046,0.1949,0.2005)\right\rangle$ & $\left\langle\left(S_{4}, 0.1\right),(0.6358,0.1634,0.2008)\right\rangle$ \\
$\vartheta_{3}$ & $\left\langle\left(S_{2}, 0.1\right),(0.4816,0.2524,0.2660)\right\rangle$ & $\left\langle\left(S_{3}, 0.1\right),(0.2769,0.1673,0.5558)\right\rangle$ \\
$\vartheta_{4}$ & $\left\langle\left(S_{3}, 0.3\right),(0.4517,0.1474,0.4008)\right\rangle$ & $\left\langle\left(S_{3}, 0.4\right),(0.2785,0.3447,0.3768)\right\rangle$ \\
$\vartheta_{5}$ & $\left\langle\left(S_{4},-0.2\right),(0.3893,0.2479,0.3628)\right\rangle$ & $\left\langle\left(S_{3}, 0.1\right),(0.3103,0.2684,0.4213)\right\rangle$ \\
\hline & $\mathbf{U}_{3}$ & $\mathbf{U}_{4}$ \\
\hline$\vartheta_{1}$ & $\left\langle\left(S_{3},-0.3\right),(0.4484,0.3231,0.2285)\right\rangle$ & $\left\langle\left(S_{3}, 0.4\right),(0.5838,0.1687,0.2474)\right\rangle$ \\
$\vartheta_{2}$ & $\left\langle\left(S_{5},-0.1\right),(0.6106,0.1477,0.2417)\right\rangle$ & $\left\langle\left(S_{6},-0.4\right),(0.7450,0.1355,0.1195)\right\rangle$ \\
$\vartheta_{3}$ & $\left\langle\left(S_{4},-0.3\right),(0.3670,0.1518,0.4812)\right\rangle$ & $\left\langle\left(S_{3},-0.2\right),(0.4533,0.2915,0.2552)\right\rangle$ \\
$\vartheta_{4}$ & $\left\langle\left(S_{3}, 0.3\right),(0.3812,0.2756,0.3432)\right\rangle$ & $\left\langle\left(S_{3}, 0.2\right),(0.5429,0.2454,0.2117)\right\rangle$ \\
$\vartheta_{5}$ & $\left\langle\left(S_{2}, 0.4\right),(0.5693,0.2600,0.1707)\right\rangle$ & $\left\langle\left(S_{2},-0.4\right),(0.5970,0.2475,0.1555)\right\rangle$ \\
\hline
\end{tabular}

Step 4. According to Table 4, we can obtain the value of the AV based on all proposed attributes by Formula (16), which is listed in Table 5.

Table 5. The value of the average solution (AV).

\begin{tabular}{cc}
\hline & Average Solution \\
\hline $\mathrm{U}_{1}$ & $\left\langle\left(S_{4},-0.4\right),(0.4526,0.0119,0.5355)\right\rangle$ \\
$\mathrm{U}_{2}$ & $\left\langle\left(S_{3}, 0.3\right),(0.4114,0.2345,0.3541)\right\rangle$ \\
$\mathrm{U}_{3}$ & $\left\langle\left(S_{3}, 0.4\right),(0.4850,0.2203,0.2947)\right\rangle$ \\
$\mathrm{U}_{4}$ & $\left\langle\left(S_{3}, 0.3\right),(0.5966,0.2096,0.1938)\right\rangle$ \\
\hline
\end{tabular}

Step 5. According to the results of the AV, we can compute the PDA and the NDA by using the Formulas (19) and (20), which are listed in Tables 6-8.

Table 6. The score values of $\vartheta^{\prime}{ }_{i j}$ and $\mathrm{AV}_{j}$.

\begin{tabular}{ccccc}
\hline & $\mathbf{U}_{\mathbf{1}}$ & $\mathbf{U}_{\mathbf{2}}$ & $\mathbf{U}_{\mathbf{3}}$ & $\mathbf{U}_{\mathbf{4}}$ \\
\hline$\vartheta_{1}$ & $\left(S_{1}, 0.2251\right)$ & $\left(S_{2},-0.4490\right)$ & $\left(S_{2},-0.3775\right)$ & $\left(S_{2}, 0.2585\right)$ \\
$\vartheta_{2}$ & $\left(S_{4}, 0.3106\right)$ & $\left(S_{3},-0.0798\right)$ & $\left(S_{3}, 0.3536\right)$ & $\left(S_{5},-0.4321\right)$ \\
$\vartheta_{3}$ & $\left(S_{1}, 0.2582\right)$ & $\left(S_{1}, 0.1069\right)$ & $\left(S_{2},-0.3834\right)$ & $\left(S_{2},-0.3047\right)$ \\
$\vartheta_{4}$ & $\left(S_{2},-0.2450\right)$ & $\left(S_{2},-0.4581\right)$ & $\left(S_{2},-0.2976\right)$ & $\left(S_{2}, 0.1565\right)$ \\
$\vartheta_{5}$ & $\left(S_{2},-0.0701\right)$ & $\left(S_{1}, 0.3958\right)$ & $\left(S_{2},-0.3356\right)$ & $\left(S_{1}, 0.1172\right)$ \\
\hline $\mathrm{AV}$ & $\left(S_{2},-0.3337\right)$ & $\left(S_{2},-0.2787\right)$ & $\left(S_{2}, 0.0080\right)$ & $\left(S_{2}, 0.3314\right)$ \\
\hline
\end{tabular}

Table 7. The results of $\mathrm{PDA}_{i j}$.

\begin{tabular}{ccccc}
\hline & $\mathbf{U}_{\mathbf{1}}$ & $\mathbf{U}_{\mathbf{2}}$ & $\mathbf{U}_{\mathbf{3}}$ & $\mathbf{U}_{\mathbf{4}}$ \\
\hline$\vartheta_{1}$ & 0.0000 & 0.0000 & 0.0000 & 0.0000 \\
$\vartheta_{2}$ & 1.5869 & 0.6965 & 0.6702 & 0.9593 \\
$\vartheta_{3}$ & 0.0000 & 0.0000 & 0.0000 & 0.0000 \\
$\vartheta_{4}$ & 0.0532 & 0.0000 & 0.0000 & 0.0000 \\
$\vartheta_{5}$ & 0.1582 & 0.0000 & 0.0000 & 0.0000 \\
\hline
\end{tabular}


Table 8. The results of $\mathrm{NDA}_{i j}$.

\begin{tabular}{ccccc}
\hline & $\mathbf{U}_{\mathbf{1}}$ & $\mathbf{U}_{\mathbf{2}}$ & $\mathbf{U}_{\mathbf{3}}$ & $\mathbf{U}_{\mathbf{4}}$ \\
\hline$\vartheta_{1}$ & 0.2648 & 0.0990 & 0.1920 & 0.0313 \\
$\vartheta_{2}$ & 0.0000 & 0.0000 & 0.0000 & 0.0000 \\
$\vartheta_{3}$ & 0.2449 & 0.3569 & 0.1949 & 0.2729 \\
$\vartheta_{4}$ & 0.0000 & 0.1043 & 0.1522 & 0.0750 \\
$\vartheta_{5}$ & 0.0000 & 0.1891 & 0.1711 & 0.5208 \\
\hline
\end{tabular}

Step 6. By calculating the values of $\mathrm{SP}_{i}$ and $\mathrm{SN}_{i}$ by Equation (23) and the attributes weighting vector $\omega=(0.22,0.36,0.28,0.14)$, we can obtain the results as

$$
\begin{gathered}
\mathrm{SP}_{1}=0.0000, \mathrm{SP}_{2}=0.9218, \mathrm{SP}_{3}=0.0000, \mathrm{SP}_{4}=0.0117, \mathrm{SP}_{5}=0.0348 \\
\mathrm{SN}_{1}=0.1520, \mathrm{SN}_{2}=0.0000, \mathrm{SN}_{3}=0.2752, \mathrm{SN}_{4}=0.0907, \mathrm{SN}_{5}=0.1889
\end{gathered}
$$

Step 7. The results of Step 6 can be normalized by Formula (24) and are listed as

$$
\begin{gathered}
\mathrm{NSP}_{1}=0.0000, \mathrm{NSP}_{2}=1.0000, \mathrm{NSP}_{3}=0.0000, \mathrm{NSP}_{4}=0.0127, \mathrm{NSP}_{5}=0.0378 \\
\mathrm{NSN}_{1}=0.4475, \mathrm{NSN}_{2}=1.0000, \mathrm{NSN}_{3}=0.0000, \mathrm{NSN}_{4}=0.6705, \mathrm{NSN}_{5}=0.3135
\end{gathered}
$$

Step 8. Based on each alternative's $\mathrm{NSP}_{i}$ and $\mathrm{NSN}_{i}$, compute the values of $\mathrm{AS}$;

$$
\mathrm{AS}_{1}=0.2238, \mathrm{AS}_{2}=1.0000, \mathrm{AS}_{3}=0.0000, \mathrm{AS}_{4}=0.3416, \mathrm{AS}_{5}=0.1756
$$

Step 9. According to the calculated results of AS, we can rank all the alternatives; the bigger the value of AS is, the better the selected alternative will be. Clearly, the rank of all alternatives is $\vartheta_{2}>\vartheta_{4}>\vartheta_{1}>\vartheta_{5}>\vartheta_{3}$, and $\vartheta_{2}$ is the best green supplier.

\subsection{Compare P2TLNs EDAS Method with Some Aggregation Operators with P2TLNs}

In this section, we compare our proposed picture 2-tuple linguistic EDAS method when using either the P2TLWA operator or the P2TLWG operator. According to the results of Table 4 and attributes weighting vector $\omega=(0.22,0.36,0.28,0.14)$, we can utilize overall $r_{i j}^{\prime}$ to $r_{i}^{\prime}$ by using the P2TLWA and P2TLWG operators, which is listed in Table 9.

Table 9. The fused values by using some picture 2-tuple linguistic number (P2TLN) aggregation operators.

\begin{tabular}{ccc}
\hline & P2TLWA & P2TLWG \\
\hline$\vartheta_{1}$ & $\left\langle\left(S_{3},-0.2\right),(0.4430,0.2737,0.2834)\right\rangle$ & $\left\langle\left(S_{3},-0.2\right),(0.4362,0.2737,0.2901)\right\rangle$ \\
$\vartheta_{2}$ & $\left\langle\left(S_{5}, 0\right),(0.6405,0.1608,0.1986)\right\rangle$ & $\left\langle\left(S_{5},-0.1\right),(0.6375,0.1608,0.2017)\right\rangle$ \\
$\vartheta_{3}$ & $\left\langle\left(S_{3}, 0\right),(0.3774,0.1926,0.4300)\right\rangle$ & $\left\langle\left(S_{2},-0.1\right),(0.3644,0.1926,0.4430)\right\rangle$ \\
$\vartheta_{4}$ & $\left\langle\left(S_{3}, 0.3\right),(0.3896,0.2561,0.3542)\right\rangle$ & $\left\langle\left(S_{3}, 0.3\right),(0.3918,0.2561,0.3520)\right\rangle$ \\
$\vartheta_{5}$ & $\left\langle\left(S_{3},-0.2\right),(0.4541,0.2585,0.2874)\right\rangle$ & $\left\langle\left(S_{3},-0.3\right)(0.4308,0.2585,0.3107)\right\rangle$ \\
\hline
\end{tabular}

According to the score function of P2TLNs, we can obtain the alternative score results which are shown in Table 10.

The ranking of alternatives by some P2TLN aggregation operators are listed in Table 11.

Comparing the results of the picture 2-tuple linguistic EDAS model using either P2TLWA or P2TLWG operators, the aggregation results are slightly different in the ranking of alternatives, and the best alternatives are the same. However, the picture 2-tuple linguistic EDAS model has the valuable characteristic of considering the conflicting attributes, and can be more accurate and effective in the application of MCGDM problems. 
Table 10. Score results of alternatives $\vartheta_{i}$.

\begin{tabular}{ccc}
\hline & P2TLWA & P2TLWG \\
\hline$s\left(\vartheta_{1}\right)$ & $\left(S_{2},-0.3905\right)$ & $\left(S_{2},-0.4158\right)$ \\
$s\left(\vartheta_{2}\right)$ & $\left(S_{4},-0.4134\right)$ & $\left(S_{4},-0.4751\right)$ \\
$s\left(\vartheta_{3}\right)$ & $\left(S_{1}, 0.4111\right)$ & $\left(S_{1}, 0.3459\right)$ \\
$s\left(\vartheta_{4}\right)$ & $\left(S_{2},-0.2719\right)$ & $\left(S_{2},-0.2649\right)$ \\
$s\left(\vartheta_{5}\right)$ & $\left(S_{2},-0.3427\right)$ & $\left(S_{2},-0.4662\right)$ \\
\hline
\end{tabular}

Table 11. Rank of alternatives by some P2TLN aggregation operators.

\begin{tabular}{cc}
\hline & Order \\
\hline P2TLWA operator & $\vartheta_{2}>\vartheta_{4}>\vartheta_{5}>\vartheta_{1}>\vartheta_{3}$ \\
P2TLWG operator & $\vartheta_{2}>\vartheta_{4}>\vartheta_{1}>\vartheta_{5}>\vartheta_{3}$ \\
P2TLNs EDAS model & $\vartheta_{2}>\vartheta_{4}>\vartheta_{1}>\vartheta_{5}>\vartheta_{3}$ \\
\hline
\end{tabular}

\section{Conclusions}

In this paper, we present the picture fuzzy EDAS model for MCGDM based on the traditional EDAS model and some fundamental theories of P2TLNs. First, we briefly reviewed the definition of P2TLNs and introduced the score function, accuracy function, and operational laws of P2TLNs. Next, to fuse the P2TLNs, we introduced some aggregation operators of P2TLNs. Furthermore, we combined the traditional EDAS model with P2TLNs, the picture fuzzy EDAS model for MCGDM was established, and the computing steps were simply depicted. Our presented model was more accurate and effective for considering the conflicting attributes. Finally, a numerical example for green supplier selection was given to illustrate this new model and some comparisons between P2TLWA and P2TLWG operators using the P2TLN EDAS model were also conducted to further illustrate advantages of the new method. In the future, the picture fuzzy EDAS model can be applied to risk analysis, MADM problems [62-65], and many other uncertain and fuzzy environments [44,66-71].

Author Contributions: S.Z., H.G., G.W., Y.W. and C.W. conceived and worked together to achieve this work, S.Z. compiled the computing work by Excel and analyzed the data, S.Z. and G.W. wrote the paper. Finally, all the authors have read and approved the final manuscript.

Funding: The work was supported by the National Natural Science Foundation of China under Grant No. 71571128 and the Humanities and Social Sciences Foundation of Ministry of Education of the People's Republic of China (16XJA630005) and the Construction Plan of Scientific Research Innovation Team for Colleges and Universities in Sichuan Province (15TD0004).

Conflicts of Interest: The authors declare no conflict of interest.

\section{References}

1. Keshavarz Ghorabaee, M.; Zavadskas, E.K.; Olfat, L.; Turskis, Z. Multi-criteria inventory classification using a new method of evaluation based on distance from average solution (EDAS). Informatica 2015, 26, 435-451. [CrossRef]

2. Wang, J.; Wei, G.W.; Lu, M. An extended VIKOR method for multiple criteria group decision making with triangular fuzzy neutrosophic numbers. Symmetry 2018, 10, 497. [CrossRef]

3. Wei, G.; Zhang, N.A. A multiple criteria hesitant fuzzy decision making with Shapley value-based VIKOR method. J. Intell. Fuzzy Syst. 2014, 26, 1065-1075.

4. Chen, N.; Xu, Z.S. Hesitant fuzzy ELECTRE II approach: A new way to handle multi-criteria decision making problems. Inf. Sci. 2015, 292, 175-197. [CrossRef]

5. Wei, G.W. Extension of TOPSIS method for 2-tuple linguistic multiple attribute group decision making with incomplete weight information. Knowl. Inf. Syst. 2010, 25, 623-634. [CrossRef]

6. Hajlaoui, S.; Halouani, N. Hesitant-fuzzy-Promethee method. In Proceedings of the 20135 th International Conference on Modeling, Simulation and Applied Optimization, Hammamet, Tunisia, 28-30 April 2013. 
7. Liao, H.C.; Xu, Z.S. Multi-criteria decision making with intuitionistic fuzzy PROMETHEE. J. Intell. Fuzzy Syst. 2014, 27, 1703-1717.

8. Li, X.Y.; Wei, G.W. GRA method for multiple criteria group decision making with incomplete weight information under hesitant fuzzy setting. J. Intell. Fuzzy Syst. 2014, 27, 1095-1105.

9. Liu, H.C.; You, J.X.; Lu, C.; Shan, M.M. Application of interval 2-tuple linguistic MULTIMOORA method for health-care waste treatment technology evaluation and selection. Waste Manag. 2014, 34, 2355-2364. [CrossRef]

10. Huang, Y.H.; Wei, G.W. TODIM method for Pythagorean 2-tuple linguistic multiple attribute decision making. J. Intell. Fuzzy Syst. 2018, 35, 901-915. [CrossRef]

11. Wang, J.; Wei, G.W.; Lu, M. TODIM method for multiple attribute group decision making under 2-tuple linguistic neutrosophic environment. Symmetry 2018, 10, 486. [CrossRef]

12. Wei, G.W. TODIM method for picture fuzzy multiple attribute decision making. Informatica 2018, 29, 555-566. [CrossRef]

13. Atanassov, K.T. Intuitionistic fuzzy sets. Fuzzy Sets Syst. 1986, 20, 87-96. [CrossRef]

14. Zadeh, L.A. Fuzzy sets. Inf. Control 1965, 8, 338-353. [CrossRef]

15. Atanassov, K.; Gargov, G. Interval valued intuitionistic fuzzy-sets. Fuzzy Sets Syst. 1989, 31, $343-349$. [CrossRef]

16. Atanassov, K.T. Operators over interval-valued intuitionistic fuzzy sets. Fuzzy Sets Syst. 1994, 64, 159-174. [CrossRef]

17. Cuong, B.C.; Kreinovich, V. Picture fuzzy sets-A new concept for computational intelligence problems. In Proceedings of the 2013 Third World Congress on Information and Communication Technologies (WICT 2013), Hanoi, Vietnam, 15-18 December 2013; pp. 1-6.

18. Singh, P. Correlation coefficients for picture fuzzy sets. J. Intell. Fuzzy Syst. 2015, 28, 591-604.

19. Son, L.H. DPFCM: A novel distributed picture fuzzy clustering method on picture fuzzy sets. Expert Syst. Appl. 2015, 42, 51-66. [CrossRef]

20. Thong, N.T.; Son, L.H. HIFCF: An effective hybrid model between picture fuzzy clustering and intuitionistic fuzzy recommender systems for medical diagnosis. Expert Syst. Appl. 2015, 42, 3682-3701. [CrossRef]

21. Thong, P.H.; Son, L.H. A novel automatic picture fuzzy clustering method based on particle swarm optimization and picture composite cardinality. Knowl. Based Syst. 2016, 109, 48-60. [CrossRef]

22. Wei, G.W. Picture fuzzy cross-entropy for multiple attribute decision making problems. J. Bus. Econ. Manag. 2016, 17, 491-502. [CrossRef]

23. Son, L.H. Measuring analogousness in picture fuzzy sets: From picture distance measures to picture association measures. Fuzzy Optim. Decis. Mak. 2017, 16, 359-378. [CrossRef]

24. Son, L.H.; Thong, P.H. Some novel hybrid forecast methods based on picture fuzzy clustering for weather nowcasting from satellite image sequences. Appl. Intell. 2017, 46, 1-15. [CrossRef]

25. Wei, G.W. Some cosine similarity measures for picture fuzzy sets and their applications to strategic decision Making. Informatica 2017, 28, 547-564. [CrossRef]

26. Wei, G.W.; Lin, R.; Wang, H.J. Distance and similarity measures for hesitant interval-valued fuzzy sets. J. Intell. Fuzzy Syst. 2014, 27, 19-36.

27. Szmidt, E.; Kacprzyk, J. A similarity measure for intuitionistic fuzzy sets and its application in supporting medical diagnostic reasoning. In Proceedings of the Artificial Intelligence and Soft Computing-ICAISC 2004, Zakopane, Poland, 7-11 June 2004; pp. 388-393.

28. Wei, G.W. Picture fuzzy aggregation operators and their application to multiple attribute decision making. J. Intell. Fuzzy Syst. 2017, 33, 713-724. [CrossRef]

29. Deng, X.M.; Wei, G.W.; Gao, H.; Wang, J. Models for safety assessment of construction project with some 2-tuple linguistic Pythagorean fuzzy Bonferroni mean operators. IEEE Access 2018, 6, 52105-52137. [CrossRef]

30. Gao, H.; Lu, M.; Wei, G.W.; Wei, Y. Some novel Pythagorean fuzzy interaction aggregation operators in multiple attribute decision making. Fundamenta Informaticae 2018, 159, 385-428. [CrossRef]

31. Li, Z.X.; Wei, G.W.; Lu, M. Pythagorean fuzzy hamy mean operators in multiple attribute group decision making and their application to supplier selection. Symmetry 2018, 10, 505. [CrossRef]

32. Wang, J.; Wei, G.W.; Wei, Y. Models for green supplier selection with some 2-tuple linguistic neutrosophic number Bonferroni mean operators. Symmetry 2018, 10, 131. [CrossRef] 
33. Wei, G.W.; Alsaadi, F.E.; Hayat, T.; Alsaedi, A. Bipolar fuzzy Hamacher aggregation operators in multiple attribute decision making. Int. J. Fuzzy Syst. 2018, 20, 1-12. [CrossRef]

34. Wei, G.W.; Gao, H.; Wang, J.; Huang, Y.H. Research on risk evaluation of enterprise human capital investment with interval-valued bipolar 2-tuple linguistic information. IEEE Access 2018, 6, 35697-35712. [CrossRef]

35. Wu, S.J.; Wang, J.; Wei, G.W.; Wei, Y. Research on construction engineering project risk assessment with some 2-tuple linguistic neutrosophic hamy mean operators. Sustainability 2018, 10, 1536. [CrossRef]

36. Wei, G.W. Some similarity measures for picture fuzzy sets and their applications. Iran. J. Fuzzy Syst. 2018, 15, 77-89.

37. Wei, G.W.; Gao, H. The generalized dice similarity measures for picture fuzzy sets and their applications. Informatica 2018, 29, 107-124. [CrossRef]

38. Wei, G.W. Picture fuzzy Hamacher aggregation operators and their application to multiple attribute decision making. Fundamenta Informaticae 2018, 157, 271-320. [CrossRef]

39. Lu, M.; Wei, G.W.; Alsaadi, F.E.; Hayat, T.; Alsaedi, A. Hesitant Pythagorean fuzzy Hamacher aggregation operators and their application to multiple attribute decision making. J. Intell. Fuzzy Syst. 2017, 33, 1105-1117. [CrossRef]

40. Wei, G.W.; Lu, M. Dual hesitant Pythagorean fuzzy Hamacher aggregation operators in multiple attribute decision making. Arch. Control Sci. 2017, 27, 365-395. [CrossRef]

41. Wu, S.J.; Wei, G.W. Pythagorean fuzzy Hamacher aggregation operators and their application to multiple attribute decision making. Int. J. Knowl. Based Intell. Eng. Syst. 2017, 21, 189-201. [CrossRef]

42. Gao, H.; Wei, G.W.; Huang, Y.H. Dual hesitant bipolar fuzzy Hamacher prioritized aggregation operators in multiple attribute decision making. IEEE Access 2018, 6, 11508-11522. [CrossRef]

43. Wei, G.W.; Alsaadi, F.E.; Hayat, T.; Alsaedi, A. Projection models for multiple attribute decision making with picture fuzzy information. Int. J. Mach. Learn. Cybern. 2018, 9, 713-719. [CrossRef]

44. Wei, G.W.; Alsaadi, F.E.; Hayat, T.; Alsaedi, A. Picture 2-tuple linguistic aggregation operators in multiple attribute decision making. Soft Comput. 2018, 22, 989-1002. [CrossRef]

45. Wei, G.W. Picture 2-tuple linguistic Bonferroni mean operators and their application to multiple attribute decision making. Int. J. Fuzzy Syst. 2017, 19, 997-1010. [CrossRef]

46. Wei, G.W. Picture uncertain linguistic Bonferroni mean operators and their application to multiple attribute decision making. Kybernetes 2017, 46, 1777-1800. [CrossRef]

47. Keshavarz Ghorabaee, M.; Zavadskas, E.K.; Amiri, M.; Turskis, Z. Extended EDAS method for fuzzy multi-criteria decision-making: An application to supplier selection. Int. J. Comput. Commun. Control 2016, 11, 358-371. [CrossRef]

48. Kahraman, C.; Keshavarz Ghorabaee, M.; Zavadskas, E.K.; Onar, S.C.; Yazdani, M.; Oztaysi, B. Intuitionistic fuzzy EDAS method: An application to solid waste disposal site selection. J. Environ. Eng. Landsc. Manag. 2017, 25, 1-12. [CrossRef]

49. Keshavarz Ghorabaee, M.; Amiri, M.; Zavadskas, E.K.; Turskis, Z. Multi-criteria group decision-making using an extended EDAS method with interval type-2 fuzzy sets. E \& M Ekonomie a Management 2017, 20, 48-68.

50. Keshavarz Ghorabaee, M.; Amiri, M.; Zavadskas, E.K.; Turskis, Z.; Antucheviciene, J. A new multi-criteria model based on interval type-2 fuzzy sets and EDAS method for supplier evaluation and order allocation with environmental considerations. Comput. Ind. Eng. 2017, 112, 156-174. [CrossRef]

51. Keshavarz Ghorabaee, M.; Amiri, M.; Zavadskas, E.K.; Turskis, Z.; Antucheviciene, J. Stochastic EDAS method for multi-criteria decision-making with normally distributed data. J. Intell. Fuzzy Syst. 2017, 33, 1627-1638. [CrossRef]

52. Peng, X.D.; Liu, C. Algorithms for neutrosophic soft decision making based on EDAS, new similarity measure and level soft set. J. Intell. Fuzzy Syst. 2017, 32, 955-968. [CrossRef]

53. Ecer, F. Third-party logistics (3PLS) provider selection via fuzzy ahp and EDAS integrated model. Technol. Econ. Dev. Econ. 2018, 24, 615-634. [CrossRef]

54. Feng, X.Q.; Wei, C.P.; Liu, Q. EDAS method for extended hesitant fuzzy linguistic multi-criteria decision making. Int. J. Fuzzy Syst. 2018, 20, 2470-2483. [CrossRef]

55. Ilieva, G. Group decision analysis algorithms with EDAS for interval fuzzy sets. Cybern. Inf. Technol. 2018, 18, 51-64. [CrossRef] 
56. Karasan, A.; Kahraman, C. A novel interval-valued neutrosophic EDAS method: Prioritization of the United Nations national sustainable development goals. Soft Comput. 2018, 22, 4891-4906. [CrossRef]

57. Keshavarz-Ghorabaee, M.; Amiri, M.; Zavadskas, E.K.; Turskis, Z.; Antucheviciene, J. A dynamic fuzzy approach based on the EDAS method for multi-criteria subcontractor evaluation. Information 2018, 9, 68. [CrossRef]

58. Stevic, Z.; Vasiljevic, M.; Zavadskas, E.K.; Sremac, S.; Turskis, Z. Selection of carpenter manufacturer using fuzzy EDAS method. Eng. Econ. 2018, 29, 281-290. [CrossRef]

59. Keshavarz-Ghorabaee, M.; Amiri, M.; Zavadskas, E.K.; Turskis, Z.; Antucheviciene, J. A comparative analysis of the rank reversal phenomenon in the EDAS and TOPSIS methods. Econ. Comput. Econ. Cybern. Stud. Res. 2018, 52, 121-134.

60. Herrera, F.; Martinez, L. The 2-tuple linguistic computational model. Advantages of its linguistic description, accuracy and consistency. Int. J. Uncertain. Fuzziness Knowl. Based Syst. 2001, 9, 33-48. [CrossRef]

61. Herrera, F.; Martinez, L. A 2-tuple fuzzy linguistic representation model for computing with words. IEEE Trans. Fuzzy Syst. 2000, 8, 746-752.

62. Wei, G.W.; Lu, M. Pythagorean fuzzy power aggregation operators in multiple attribute decision making. Int. J. Intell. Syst. 2018, 33, 169-186. [CrossRef]

63. Wei, G.W.; Wei, Y. Similarity measures of Pythagorean fuzzy sets based on the cosine function and their applications. Int. J. Intell. Syst. 2018, 33, 634-652. [CrossRef]

64. Wei, G.W.; Zhang, Z.P. Some single-valued neutrosophic Bonferroni power aggregation operators in multiple attribute decision making. J. Ambient Intell. Humaniz. Comput. 2019, 10, 863-882. [CrossRef]

65. Wei, G.W. Pythagorean fuzzy Hamacher power aggregation operators in multiple attribute decision making. Fundamenta Informaticae 2019, 166, 57-85.

66. Wang, J.; Wei, G.W.; Gao, H. Approaches to multiple attribute decision making with interval-valued 2-tuple linguistic Pythagorean fuzzy information. Mathematics 2018, 6, 201. [CrossRef]

67. Wei, G.; Wei, Y. Some single-valued neutrosophic dombi prioritized weighted aggregation operators in multiple attribute decision making. J. Intell. Fuzzy Syst. 2018, 35, 2001-2013. [CrossRef]

68. Hashemkhani Zolfani, S.; Saparauskas, J. New application of SWARA method in prioritizing sustainability assessment indicators of energy system. Eng. Econ. 2013, 24, 408-414.

69. Wang, J.; Gao, H.; Wei, G.W.; Wei, Y. Methods for multiple-attribute group decision making with q-rung interval-valued orthopair fuzzy information and their applications to the selection of green suppliers. Symmetry 2019, 11, 56. [CrossRef]

70. Wang, R.; Wang, J.; Gao, H.; Wei, G.W. Methods for MADM with picture fuzzy muirhead mean operators and their application for evaluating the financial investment risk. Symmetry 2019, 11, 6. [CrossRef]

71. Sarkar, B.; Mahapatra, A.S. Periodic review fuzzy inventory models with variable lead time and fuzzy demand. Int. Trans. Op. Res. 2017, 24, 1197-1227. [CrossRef] 